\title{
Consequences of Disrupting Salmonella AI-2 Signaling on Interactions Within Soft Rots
}

\author{
Clayton E. Cox, Michael McClelland, and Max Teplitski
}

First and third authors: Interdisciplinary Ecology Program, Department of Soil and Water Science, University of Florida-Institute of Food and Agricultural Sciences, Gainesville 32611; and second author: Department of Pathology, University of California, Irvine 92697-4800. Accepted for publication 27 November 2012.

\section{ABSTRACT}

Cox, C. E., McClelland, M., and Teplitski, M. 2013. Consequences of disrupting Salmonella AI-2 signaling on interactions within soft rots. Phytopathology 103:352-361.

Within soft rots, Salmonella spp. reach population densities 10- to 100fold higher than within intact plants. The hypothesis that Salmonella spp. exchange AI-2 signals with Pectobacterium carotovorum to increase its competitive fitness was tested using mutants involved in AI-2 production (luxS) or perception (lsrACDBF or $l s r G$ ). Co-infections of a wild-type Salmonella sp. and its AI-2 mutants (at $\approx 3$ to $10^{4}$ ) were established in green or red tomato ('FL 47' or 'Campari' for 3 or 5 days) as well as tomato co-infected with Pectobacterium (at $10^{9}$ ) or its luxS mutant. There were no significant differences in the competitive fitness of Salmonella, indicating that $\mathrm{AI}-2$ signaling is not a major input in the interactions between these organisms under the tested conditions. A Salmonella lsrG::tnpR-lacZ resolvase in vivo expression technology (RIVET) reporter, constructed to monitor AI-2-related gene expression, responded strongly to the luxS deletion but only weakly to external sources of AI-2. Growth in soft rots generally decreased RIVET resolution; however, the effect was not correlated to the luxS genotype of the Pectobacterium sp. The results of this study show that AI-2 signaling offers no significant benefit to Salmonella spp. in this model of colonization of tomato or soft rots.

Additional keywords: produce safety, quorum sensing.
Salmonella spp. contamination of produce is responsible for an increasing number of foodborne outbreaks (19). Raw tomato fruit are a prominent produce vector of salmonellosis and have been linked to at least 12 multi-state outbreaks of salmonellosis since 1990 (1,11). Marketing surveys and laboratory studies establish a clear link between the composition of plant-associated microbiota and Salmonella spp. persistence in and on plants $(2,27,63)$. Salmonella spp. are able to integrate into multicellular consortia formed by epiphytes on leaf surfaces and benefit from damage induced by phytopathogens, reaching higher densities when growing within bacterial lesions on fruit and leaves $(2,7,8)$. Market produce with visual soft-rot symptoms was twice as likely to harbor Salmonella spp. and harbored the pathogen at levels more than a log-fold higher than asymptomatic or mechanically damaged produce (63). In a Salmonella enterica-Pectobacterium carotovorum co-infection model, a soft rot caused by $P$. carotovorum increases $S$. enterica proliferation up to three-log-fold in green market tomato (36). Although the beneficial association between human enteric pathogens and phytobacteria is well established and may ultimately determine the safety of fresh produce, the mechanisms governing these interactions are currently unknown.

It is hypothesized that human enteric pathogens specifically benefit from the plant polymer-degradative abilities of pectinolytic bacteria, such as $P$. carotovorum, which are normal members of plant-associated microbial communities, to gain access to protected environments or increased availability of nutrients $(6,20,21$, 45,61,63). On lettuce, Escherichia coli O157:H7 preferentially colonizes damaged surfaces and reaches a 27 -fold higher population size when grown on leaves infected with Dickeya dadantii (formerly Erwinia chrysanthemi) 3937 (7,66). However, when

Corresponding author: C. E. Cox; E-mail address: cec9@ufl.edu

http://dx.doi.org/10.1094/PHYTO-09-12-0237-FI

(c) 2013 The American Phytopathological Society grown on lettuce leaves infected with $D$. dadantii out $C$ mutant, which is completely deficient in the export of cell-wall-degrading enzymes, including pectate lyases (PELs), the ultimate population size of Escherichia coli $\mathrm{O} 157: \mathrm{H} 7$ is indistinguishable from that reached when grown on non- $D$. dadantii-infected leaves (66).

$P$. carotovorum is a broad-host-range pectinolytic phytopathogen, which utilizes quorum sensing (QS) systems to coordinate expression of the genes involved in the production of hydrolytic exoenzymes required for virulence in plants $(12,15,28,41)$. There are two well-characterized population-density-dependent gene regulatory QS systems in these bacteria: one based on the production and perception of $\mathrm{N}$-acyl homoserine lactone (AHL) signals and a second which utilizes the LuxS-dependent autoinducer (AI)-2 signal. The role of these QS systems in mediating interactions within plant-associated microbial communities is well established $(5,9,38,58,62,65)$. Because many enteric and phytobacteria share components of QS signaling pathways, it has been commonly hypothesized that signal exchange plays a major role in mediating interactions in planta and could specifically empower beneficial interactions between Salmonella spp. and soft-rot-causing organisms in plant tissues.

By exploiting these QS signals, S. enterica may be able to increase the suitability of the produce environment and induce its own favorable genetic regulatory changes. $P$. carotovorum possess a fully functional AHL system which is required for plant virulence. Salmonella spp. lack an AHL synthase but possess an orphan, yet functional AHL receptor, encoded by sdiA, which allow them to perceive AHL signals and differentially regulate expression of several genes with yet-unknown functions (49). Although $S$. enterica can respond to AHL signals produced by $P$. carotovorum as well as their chemical homologs in rich laboratory media, it is unable to recognize AHLs in planta because expression of $s d i A$ is "off" within tomato soft rots $(36,47)$. Furthermore, the fitness of a Salmonella sdiA mutant was not affected within soft rots, indicating that the ability of Salmonella spp. to interact with phytobacteria under normal environmental condi- 
tions does not depend on AHL- or SdiA-mediated signal exchange under the experimental conditions used in the study (36).

Given the wide distribution of the luxS synthase gene, AI-2mediated QS was originally believed to be a common method for interspecies communication (46). This view has been somewhat tempered by evidence showing a relative rarity of AI- 2 receptors or response regulators although, among enterobacteriaceae, receptors are more common among pathogenic and endosymbiotic species $(17,43,44,50)$. Both Salmonella spp. and $P$. carotovorum are known to possess functional AI-2 signaling systems, making interspecies communication feasible. Therefore, with this study, we tested the hypothesis that AI-2-mediated signaling contributes to the fitness of $S$. enterica sv. Typhimurium within soft rots caused by $P$. carotovorum on tomato.

The rationale for this hypothesis is provided by reports that normal microbiota recovered from several types of fresh produce were positive for AI-2-like activity, and rinses taken from the surface of tomato fruit were able to enhance biofilm formation of an E. coli luxS mutant in polystyrene plates, suggestive of AI-2 activity (29). AI-2 activity is known to peak in late exponential phase before declining into stationary phase in both species, which could be an indication of conserved regulatory mechanisms $(15,51)$. Interruption of AI-2 signaling in $P$. carotovorum delays production of virulence factors, including PEL, at low population densities, and luxS-deficient strains are less virulent in potato $(15,28)$. Salmonella luxS mutants show differential regulation of virulence phenotypes in vitro and in vertebrate hosts $(8,14,25,42$, 64). However, in Salmonella spp., the only known target of AI-2 is the lsrACDBFG operon, whose sole known function is AI-2 uptake and processing (56). The $l s r A C D B F G$ operon and the divergent $l s r R$ are controlled by a bidirectional promoter region upstream of $l s r A$. Typically, LsrR acts as a repressor, blocking the lsrA promoter until AI-2 reaches a sufficient intracellular concentration to relive repression by LsrR and allow transcription of lsrACDBFG.

Little is known about the role of AI-2 signaling in enteric bacteria during colonization of soft rots. A Salmonella luxS mutant has no affect on colonization of cilantro leaves and neither the mutant nor wild-type Salmonella sp. benefits from co-colonization of cilantro with AI-2-producing epiphytes (8). However, only a signal synthase (luxS) mutant was tested in that study, opening the possibility that the production of AI-2 by the plant's native epiphytes complemented the signaling defect in trans. Furthermore, Salmonella luxS activity is known to increase in response to the rich environment, acidic $\mathrm{pH}$, and high osmolarity of the human gut (51). These conditions are not present on plant surfaces but are similar to those encountered in tomato fruit, which may represent an environment suitable for Salmonella AI-2 activity. The goal of this study was to determine the contribution of AI-2 signaling to the fitness of Salmonella spp. in market tomato as well as in tomato with $P$. carotovorum soft rots. For this study, competitive fitness was defined as the relative ability of a particular strain to multiply to a higher population density compared with the isogenic wild-type strain and was evaluated using defined $l u x S$ and $l s r$ operon mutants. Resolvase in vivo expression technology (RIVET) reporters within the corresponding promoters were also used to evaluate the activity of AI-2-related genes within the tomato environment.

\section{MATERIALS AND METHODS}

Strains and culture conditions. Salmonella and E. coli strains were grown at $37^{\circ} \mathrm{C}$ and Pectobacterium strains at $30^{\circ} \mathrm{C}$ in LuriaBertani (LB) medium, unless noted, with antibiotics as necessary. Overnight cultures of Pectobacterium SCC3193 and SCC6023 for use in tomato infections were grown in LB $0.2 \%$ (wt/vol) glucose. Without pre-growth in a glucose-containing medium, these strains induced soft rots unreliably. This step was not necessary for
Pectobacterium SR38, a strain originally isolated as hypervirulent in tomato (3). Antibiotics were used at the following final concentrations: ampicillin $(200 \mu \mathrm{g} / \mathrm{ml})$, kanamycin $(50 \mu \mathrm{g} / \mathrm{ml})$ tetracycline $(10 \mu \mathrm{g} / \mathrm{ml})$, and chloramphenicol $(10 \mu \mathrm{g} / \mathrm{ml})$. All strains used in this study are listed in Table 1.

Mutant construction. Deletion mutants were constructed using the methods described by Datsenko and Wanner (16) by removing the portion of the gene between the stop and start codons. RIVET reporters were constructed using the previously described methods as adapted for Salmonella spp. $(13,31,37)$. S. enterica sv. Typhimurium 14028 was used as the host for all deletion mutants and its derivative JS246 as the host for all RIVET reporters. Primers used are listed in Table 1 . The $l s r G$ RIVET reporter (CEC0015) was constructed via integration of pGOA1193 containing an $l s r$ operon fragment starting $234 \mathrm{bp}$ upstream of $l s r G$ (located in $l s r F$ ) and ending $24 \mathrm{bp}$ upstream of the putative location of the lsrACDBFG operon transcriptional terminator (primers cec045 and cec046), as inferred by homology to E. coli. This recombination results in a merodiploid generating an $l s r G:: \operatorname{tnpR}$-lacZ fusion along with the duplication of an intact $l s r G, \approx 7,000$ bp downstream of the insertion site. The insertion was confirmed with primers cec047 and MT59. As the last gene in the $l s r$ operon, the lsrG::tnpR-lacZ reporter should record all activity driven via the lsrACDBFG operon promoter, $\mathrm{P}_{l s r A}$, with minimal disruption to the operon. Additionally, microarray evidence from $E$. coli shows that $l s r G$ is highly activated by $l u x S$ in stationary phase, suggesting that the locus is an ideal location for a reporter (60). Reporter strain CEC0018 was constructed by transducing $4 l u x S 51:: F R T-$ kanR-FRT from MM_019-C10 into CEC0015 via phage P22mediated generalized transduction. A $\mathrm{P}_{\text {luxs }}$-tnpR-lac $Z$ reporter (CEC0026) was constructing using $\lambda$-red recombination to replace all of $\operatorname{luxS}$, including the start and stop codons (primers cec111 and cec112), with FRT-kanR-FRT amplified from plasmid pKD4. Primers cec050 and cec051 were used to confirm the insertion or kanR as well as its removal via plasmid pCP20. The resulting FRT scar was utilized as an integration site for the suicide plasmids pCE70 or pCE71, creating a fusion of the luxS promoter and tnpR-lacZ. Following the electroporation of pCE70 or pCE71, transformants were screened with primers cec113 and BA184 to select those in which the pCE7x plasmid integrated in the desired orientation.

Confirmation of AI-2 production via the Vibrio harveyi LUX assay. The production of AI-2 by the $S$. enterica and P. carotovorum strains used in this study was confirmed using the Vibrio harveyi BB170 reporter assay, as previously described $(4,54)$. To prepare Salmonella and Pectobacterium culture filtrates, overnight cultures were washed three times in phosphate-buffered saline (PBS) and diluted 1/100 into fresh LB without antibiotics. Subcultures were harvested at an optical density at $600 \mathrm{~nm}\left(\mathrm{OD}_{600}\right)$ of $\approx 1.00$ by centrifuging $1 \mathrm{ml}$ of culture at $13,000 \times g$ for $1 \mathrm{~min}$ to pellet cells. The resulting supernatant was sterilized by passing through a $0.22-\mu \mathrm{m}$ filter. Recovered culture filtrates were stored at $-20^{\circ} \mathrm{C}$ until use.

Competitive fitness assays. The competitive fitness of defined deletion mutants compared with wild-type $S$. enterica sv. Typhimurium 14028 was determined using a competitive index, as described previously (36). Briefly, unwaxed red ripe market tomato fruit ('Campari', grown hydroponically and purchased at local grocery stores) or unwaxed mature green tomato fruit ('Florida 47' [FL47], grown conventionally and obtained directly from processors or harvested in the field) were wounded three times by piercing the skin with flame-sterilized paper clips. Care was taken to wound the tomato between the seed sacks for a more uniform infection. For soft-rot treatments, $3 \mu \mathrm{l}$ of a Pectobacterium suspension $\left(10^{9} \mathrm{CFU} / \mathrm{ml}\right)$ was introduced into each wound and allowed to infiltrate the tomato tissue. Next, $3 \mu$ l of a roughly 50:50 mix of the wild-type and mutant Salmonella $\left(10^{4} \mathrm{CFU} / \mathrm{ml}\right)$ was introduced to each wound. S. enterica sv. Typhimurium 
14028 and its isogenic tetracycline-resistant derivative JS246 were similarly inoculated as a control. Prior to the infections, all overnight cultures were washed three times and resuspended in PBS. A sample of each inoculum was plated on xylose lysine deoxycholate agar (XLD) and 50 individual colonies were patched to LB kanamycin or tetracycline plates to determine the initial mutant/wild-type ratio. All tomato fruit were incubated at $22^{\circ} \mathrm{C}$ for 3 days for green fruit and 5 days for red fruit. These incubation times were sufficient for development of full soft-rot symptoms in fruit inoculated with Pectobacterium. Salmonella were recovered directly from the wound using a flame-sterilized wire loop cooled in sterile PBS, quad streaked onto XLD, and then incubated at $42^{\circ} \mathrm{C}$ to limit the growth of phytobacteria. Severely rotted tomato fruit were harvested by stomaching in a Whirl-Pak bag (Nasco, Fort Atkinson, WI) along with $50 \mathrm{ml}$ of PBS at $260 \mathrm{rpm}$ in a stomacher for $1 \mathrm{~min}$. An aliquot of the resulting homogenate was plated on XLD and then incubated at $42^{\circ} \mathrm{C}$. After overnight growth, 50 individual colonies were patched to LB plates with appropriate antibiotics to count the number of antibiotic-resistant mutant colonies. Shifts in the mutant/wild-type ratio between the inoculum and recovered samples were used to calculate a competitive index (CI) according to the following equation: $\mathrm{CI}=\left[\mathrm{M}_{\text {out }} / \mathrm{WT}_{\text {out }}\right] /$ $\left[\mathrm{M}_{\mathrm{in}} / \mathrm{WT}_{\mathrm{in}}\right]$, where $\mathrm{M}$ is the number of mutant cells and WT is the number of the wild-type cells in the inoculum (in) or in the recovered samples (out). The CI values were log transformed to allow even comparison between increases and decreases in competitive fitness.

Response of the $l s r G$-tnpR reporter to exogenous AI-2. Both LacZ activity and RIVET resolution of strain CEC0018 (lux $S^{-}$) were used to test for perception of exogenous AI-2 by the
lsrG::tnpR-lacZ reporter. Strain CEC0015 was used as a positive control for $\operatorname{lux} S^{+}$expression levels. Synthetic (S)-4,5-dihydroxy2,3-pentandione (DPD), the universal AI-2 precursor, was supplied as a $3.7-\mathrm{mM}$ stock solution from OMM Scientific (Dallas, TX) and stored at $-20^{\circ} \mathrm{C}$ until use. Prior to assays, overnight cultures of the reporters were started from glycerol stocks in LB with antibiotics. Cells were washed to remove tetracycline and diluted 1/100 into fresh LB without antibiotics. DPD was used at a final concentration of $10 \mu \mathrm{M}$ and added to the medium just prior to inoculation of the assay or after $2 \mathrm{~h}$ of growth. Other authors have indicated that incubation with $10 \mu \mathrm{M}$ DPD is sufficient to generate strong activation of previously reported $l s r$ operon reporters $(18,33,55)$. Assays were performed at $37^{\circ} \mathrm{C}$ in $10 \mathrm{ml}$ of LB. Samples were taken at $0,2,4$, and $8 \mathrm{~h}$ for analysis of LacZ activity and at $24 \mathrm{~h}$ for analysis of TnpR resolution. LacZ activity was determined using a modified Miller assay based on the adapted 96-well protocol of Griffith and Wolf $(22,32)$. Plates were analyzed using a multimode microtiter plate reader (Victor3; Perkin Elmer, Fremont, CA), equipped with Wallac1420 Manager Work-station software. Modified Miller units were calculated as follows: modified Miller units $=\left[1,000 \times\right.$ absorbance $\left._{405}\right] /\left[t_{\min } \times\right.$ sample volume $\left.\mathrm{ml} \times \mathrm{OD}_{600}\right]$. At $24 \mathrm{~h}$, an aliquot of the culture was streaked onto XLD at $37^{\circ} \mathrm{C}$ using a sterile wire loop. After overnight growth, 50 colonies were patched to $\mathrm{LB}$ tetracycline plates which were incubated overnight at $37^{\circ} \mathrm{C}$. The resolution was determined as the number of the tetracycline-sensitive colonies divided by the total number of patched colonies.

In vitro reception of Pectobacterium AI-2 signaling by Salmonella spp. The Salmonella lsrG::tnpR-lacZ RIVET reporter strains CEC0015 and CEC0018 were used to test whether the

TABLE 1. Bacterial strains, plasmids, and primers used in this study

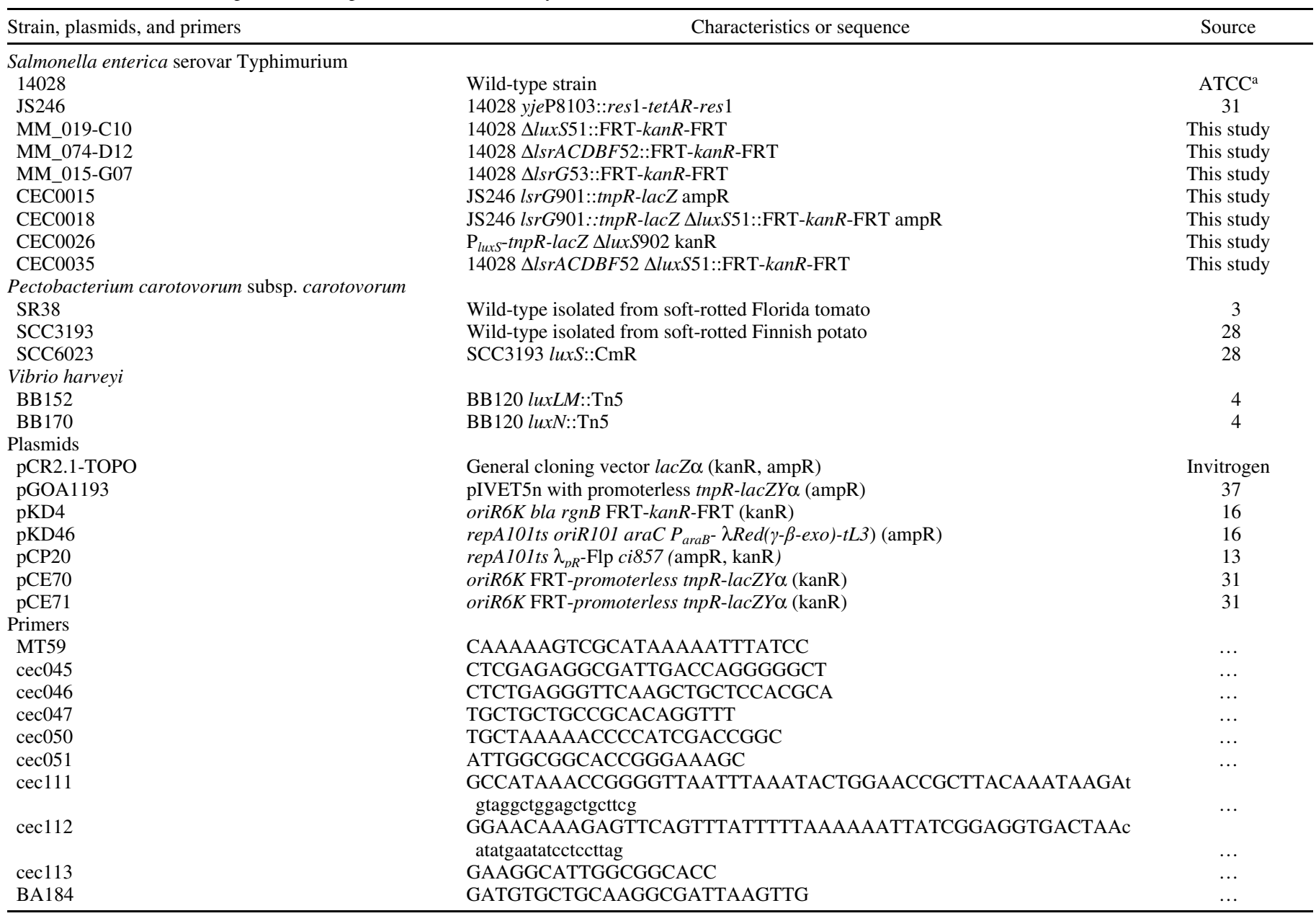

a American Type Culture Collection. 
perception of AI-2 produced by the $P$. carotovorum strains SR38, SCC3193, or SCC6023 was at sufficient levels to drive Salmonella AI-2-dependent gene expression during co-cultures in soft agar. Both $S$. enterica CEC0018 and $P$. carotovorum SCC6023 lack the $\operatorname{luxS}$ synthase and are unable to produce their own AI-2. Overnight reporter cultures were washed three times in sterile PBS to remove antibiotics and diluted $1 / 10,000$. The inoculum was mixed $1 / 100$ into $7 \mathrm{ml}$ of warm LB $0.3 \%$ soft agar, poured over the previously prepared $1.5 \%$ agar plates, and allowed to set in a sterile flow hood. Plates were incubated at $22^{\circ} \mathrm{C}$ and sampled at $24 \mathrm{~h}$. Samples were recovered with a sterile wire loop directly from the soft agar and streaked to XLD plates incubated at $37^{\circ} \mathrm{C}$ overnight to select for Salmonella spp. RIVET resolution was then quantified as above.

In vivo promoter expression measured via RIVET assays. The RIVET reporters CEC0015, CEC0018, and CEC0026 were used to examine the activity of the $\operatorname{luxS}$ promoter and the $l s r$ operon during the colonization of intact and soft-rotted green tomato fruit. Tomato infections were prepared in the same manner as for the competition assays, except each wound was inoculated with $3 \mu \mathrm{l}$ of $10^{4} \mathrm{CFU} / \mathrm{ml}$ dilutions of the RIVET reporter. The tomato fruit were incubated at $22^{\circ} \mathrm{C}$ for 2 days, which was sufficient for $\geq 20$-mm soft-rot lesions to appear. Salmonella spp. were recovered directly from the wound using a flame-sterilized wire loop cooled in sterile PBS and quad streaked on XLD antibiotic plates incubated at $37^{\circ} \mathrm{C}$.

Pectate lyase assay. The pectate lyase (PEL) activity of Pectobacterium SR38 was measured according to a modified version of the assay described by Matsumoto et al. (30). Briefly, SR38 was grown overnight in M9 with $0.4 \%$ glycerol and diluted $1 / 100$ into M9 with $1.0 \%$ citrus pectin (Sigma-Aldrich, St. Louis) at pH 6.8. The subculture was grown for $8 \mathrm{~h}$ at $30^{\circ} \mathrm{C}$ and the $\mathrm{OD}_{600}$ recorded with a spectrophotometer (BioSPEC-mini, Shimadzu, Japan). A $1-\mathrm{ml}$ aliquot was centrifuged at $16,000 \times g$ for $3 \mathrm{~min}$ and $10 \mu \mathrm{l}$ of the supernatant removed. The supernatants were mixed with 990 $\mu \mathrm{l}$ of PEL assay buffer (0.1 M Tris- $\mathrm{HCl}$ [pH 8.5], $0.1 \mathrm{mM} \mathrm{CaCl}_{2}$, and $0.05 \%$ [wt/vol] sodium polypectate [M. Burger Enterprises, Madison, WI]) which was prewarmed to $30^{\circ} \mathrm{C}$. The suspension was incubated at $30^{\circ} \mathrm{C}$ for $15 \mathrm{~min}$ before the $\mathrm{OD}_{230}$ was recorded with a spectrophotometer. The $\mathrm{OD}_{230}$ was rerecorded after an additional $10-\mathrm{min}$ incubation at $30^{\circ} \mathrm{C}$. One unit of PEL activity was defined as a 0.001 increase in $\mathrm{OD}_{230}$ per minute. Salmonella 14028 culture filtrates $(1 \% \mathrm{vol} / \mathrm{vol})$, DPD $(10 \mu \mathrm{M})$, and $1 / 100$ dilutions of Salmonella spp. were added to the SR38 culture $4 \mathrm{~h}$ into the incubation. The Salmonella strains were grown overnight in $\mathrm{LB}$ at $37^{\circ} \mathrm{C}$ and washed $3 \times$ in $\mathrm{PBS}$ to remove antibiotics prior to use.

Data analysis. Box plots, analysis of variance (ANOVA), pairwise Student's $t$ test, Dunnett's $t$ test, and the Tukey-Kramer honestly significant difference (HSD) method were generated using JMP 9.0 (SAS Institute, Cary, NC). Dunnett's $t$ test was used to compare competitive co-infections in intact tomato fruit with the JS246 versus 14028 control. A two-tailed Student's $t$ test with unequal variances was performed in Excel to compare similar competitive co-infections between tomato treatments; $\alpha=0.05$ for all tests.

\section{RESULTS}

AI-2 activity of wild-type and mutant strains. Analysis of AI-2 activity using the $V$. harveyi BB170 reporter assay showed high levels of activation from $0.4 \mu \mathrm{M}$ DPD, culture filtrates collected from the lux $S^{+}$strains of Pectobacterium, and $l u x S^{+}$ strains of Salmonella grown at $22^{\circ} \mathrm{C}$ (Fig. 1). Interestingly, the culture filtrates of the same strains of Salmonella grown at $37^{\circ} \mathrm{C}$ only activated the $V$. harveyi reporter $\approx 10$-fold (at least 10 -fold less than the culture filtrates harvested from the cultures grown at $22^{\circ} \mathrm{C}$ ). In both species, an $\operatorname{luxS}$ mutation essentially eliminated detectable AI-2 activities in the supernatant, consistent with the function of LuxS in AI-2 synthesis (52). The supernatant of the hypervirulent $P$. carotovorum SR38 produced approximately double the AI-2 activity of the laboratory strain SCC3193 (Fig. 1). Whether or not this elevated production of AI-2 is linked to the hypervirulence of SR38 is not known and was not tested with this study.

Fitness of Salmonella luxS and lsr operon mutants in soft rots. The fitness of defined AI-2 Salmonella mutants was tested under several conditions in order to examine the effect that AI-2 signal production or perception, growth in two common market cultivars, or the presence of soft rot has on the proliferation of Salmonella spp. in tomato (Fig. 2). None of the mutations tested under any of the conditions, intact green tomato $(F=1.206, P=$ $0.321)$, green tomato with soft rots caused by $P$. carotovorum SR38 $(F=1.537, P=0.203)$, intact red tomato $(F=1.537, P=$ $0.203)$, or red tomato with SR38 soft rots $(F=1.234, P=0.309)$ were determined to be significant by ANOVA, indicating that

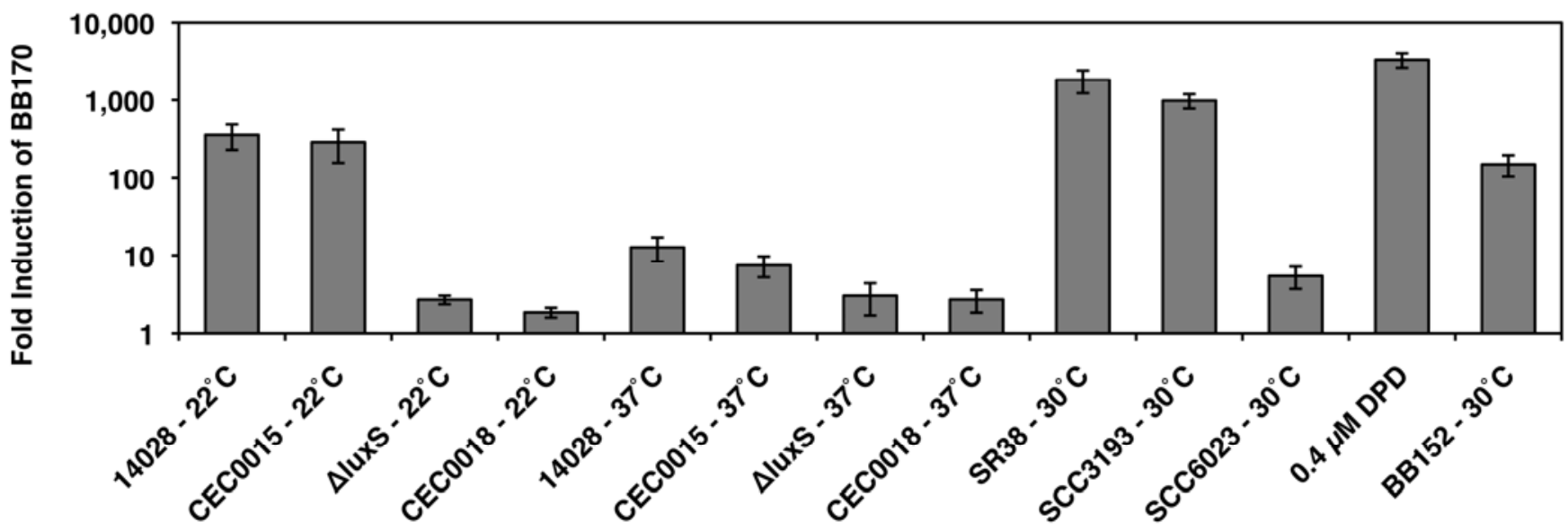

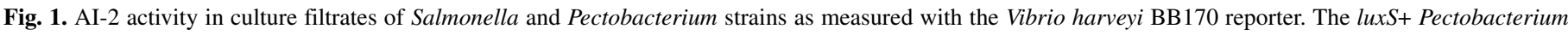

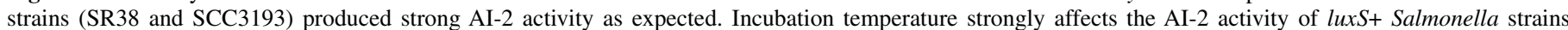

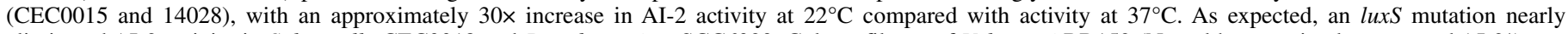

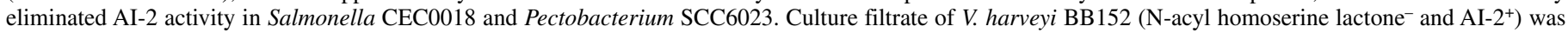

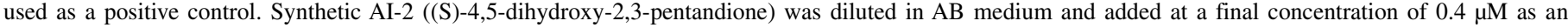

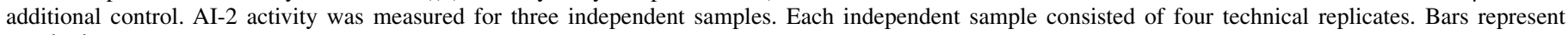
standard error. 
none of the Salmonella mutations significantly altered the competitive fitness compared with the wild type. Overall, the majority of the mean $\log$ CI values fell between -0.25 and 0.25 . Previous studies have considered only a CI of $\geq 3$ to be biologically relevant $(\log 3=|0.477|)(48)$. Only two assays, luxS versus 14028 in SR38 soft-rotted green tomato $(\log C I=0.491)$ and luxS lsrACDBF versus 14028 in SR38 soft-rotted red tomato $(\log C I=0.695)$ exceed this arbitrary threshold. However, the Tukey-Kramer HSD confirmed that these increases were not significant.

In comparing the mean $\log C$ I results for each mutant between treatments, growth in SR 38 soft rots noticeably increased the competitive fitness of the luxS mutant $(\Delta \log C I=0.624, P=$ $0.070)$ in green tomato and both the luxS mutant $(\Delta \log C I=0.431$, $P=0.223)$ and luxS lsrACDBF double mutant $(\triangle \log C \mathrm{I}=0.386$, $P=0.274)$ in red tomato. However, the effects due to SR38 soft rots were not significant as determined by Student's $t$ test.

Interestingly, the luxS mutant showed a trend of decreased fitness while the luxS lsrACDBF double mutant showed a trend of increased fitness in red Campari tomato compared with green FL47 for both intact and soft-rotted samples. However, due to differences in cultivar and incubation times, the effect of ripeness stage could not be analyzed.

Effect of Pectobacterium luxS genotype on the fitness of Salmonella luxS and $l s r$ operon mutants in soft rots. Competitive fitness assays in green tomato fruit soft rotted by either wild-type (SCC3193) or luxS ${ }^{-}$(SCC6023) Pectobacterium strains showed that both the Salmonella luxS and luxS lsrACDBF mutants were more competitive than the wild type only in the soft rots caused by Pectobacterium SCC6023 (Fig. 3). The increase in fitness for the Salmonella luxS mutant was significant by ANOVA $(F=3.706, P=0.038)$; however, no pairs were significant when analyzed with the more conservative Tukey-Kramer HSD test
$(P=0.054$, intact green tomato versus SCC6023 soft rot and $P=$ 0.070 , SCC3193 soft rot versus SCC6023). The increase in fitness for the luxS lsrACDBF double mutant was not significant by ANOVA $(F=2.925, P=0.069)$ and, similarly, no pairs were significant according to the Tukey-Kramer HSD test $(P=0.266$, intact green tomato versus SCC6023 soft rot and $P=0.060$, SCC3193 soft rot versus SCC6023).
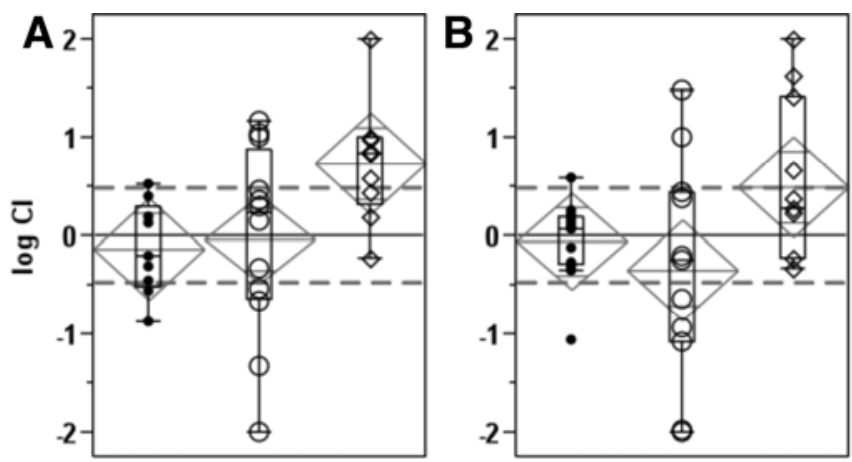

Fig. 3. Effect of Pectobacterium luxS genotype on the Salmonella AI-2 mutants A, MM_074-D12 (luxS-) and B, CEC0035 (lsrACDBF-luxS-). The fitness of the Salmonella mutants in non-soft-rotted $(\bullet)$ or Pectobacterium SCC3193 (luxS+, ○) or Pectobacterium SCC6023 (luxS-, $\diamond)$ soft-rotted green tomato fruit was compared using the Tukey Kramer honestly significant difference test. No results were significant. Box plots (represented by rectangles) show 10, 25, 75, and 90\% quantiles. Points outside the whisker lines are treated as outliers. Gray diamonds represent analysis of variance, where the middle line is the mean and the upper and lower lines show the $95 \%$ confidence intervals. Dashed gray lines correspond to log competitive index $=|0.477|$, the threshold for differences in competitive fitness considered to be biologically relevant. The zero line indicates no difference in competitive fitness.
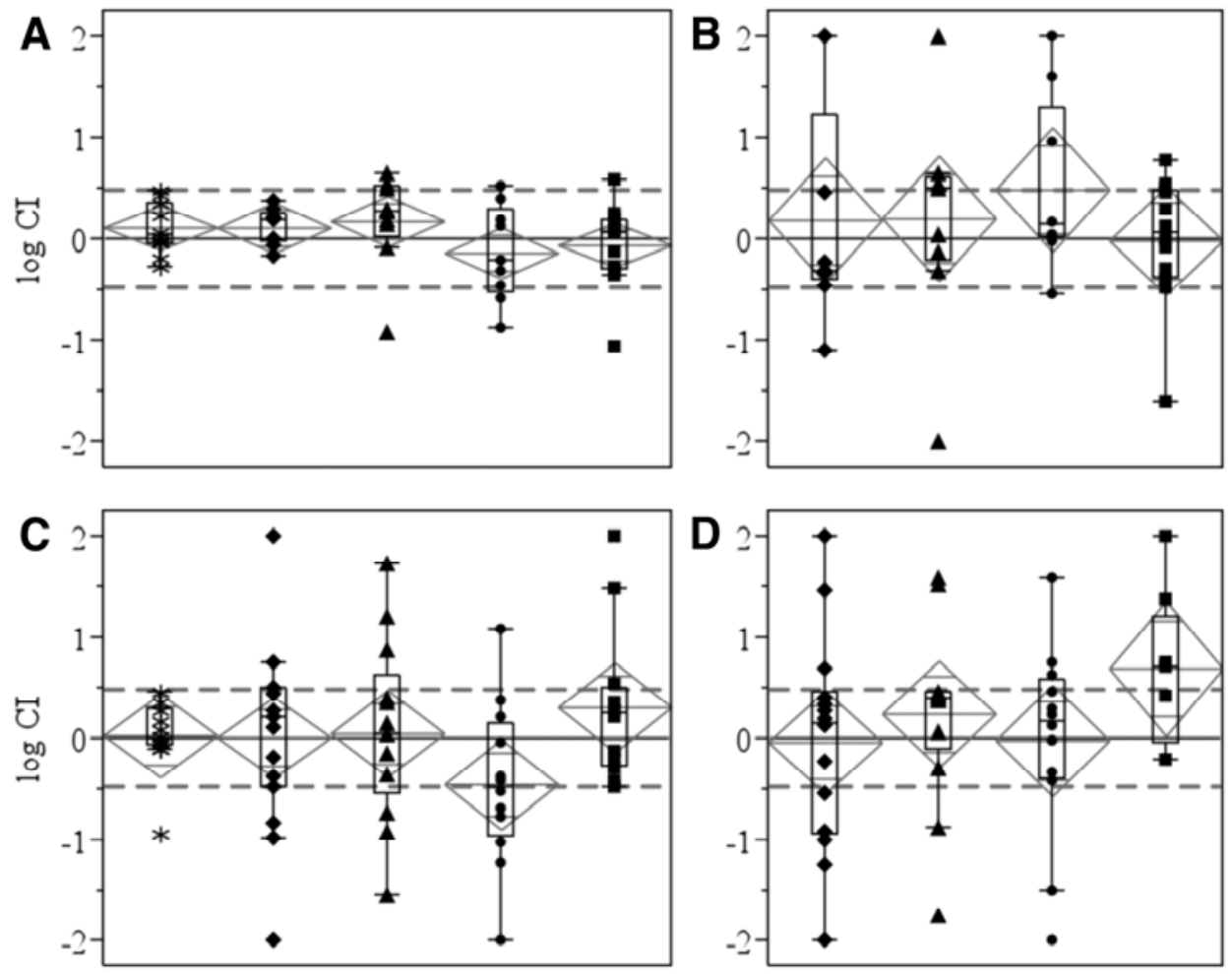

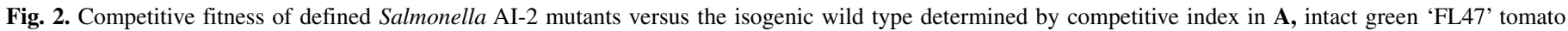

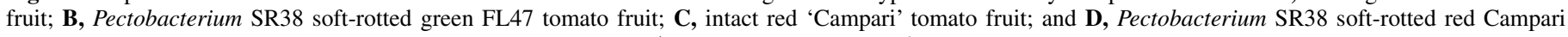

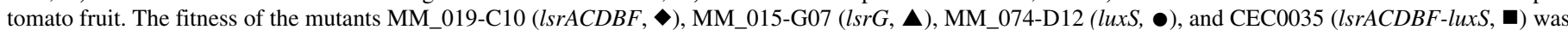

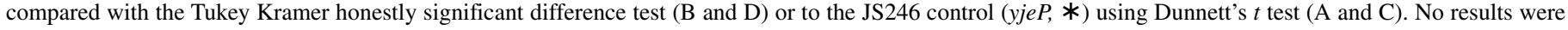

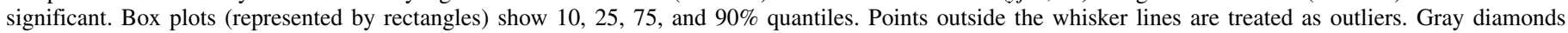

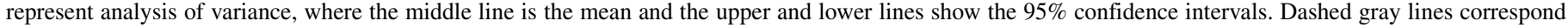

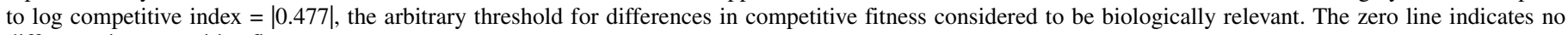
difference in competitive fitness. 
Response of $\boldsymbol{l s r} G$-tnpR to synthetic AI-2. Because of the lack of effect on fitness observed for the Salmonella AI-2 mutants, the ability of the $l s r$ operon to respond to exogenous AI-2 was examined using RIVET reporters. The lsrG::tnpR-lacZ reporter was constructed in $\operatorname{lux} S^{+}$and $\operatorname{luxS} S^{-}$backgrounds to distinguish between responses to the $\operatorname{luxS}$ genotype and exogenous AI-2 signal. Response to synthetic AI-2 was tested in LB liquid culture at $37^{\circ} \mathrm{C}$; however, the addition of $10 \mu \mathrm{M}$ DPD, the synthetic AI-2 precursor, resulted in only a small increase in activity and failed to rescue the $\operatorname{luxS} S^{-}$reporter back to wild-type levels (Fig. 4). Results of the RIVET assay showed that the reporter responded strongly to the luxS genotype but only weakly to the presence of exogenous AI-2. The results of the Miller assay showed that a relatively small increase in LacZ activity, $<2 \times$, generated a large (40x) difference in TnpR-based resolution. Because it is based on enzymatic activity, the Miller assay allows for a continuous reporter response, whereas RIVET requires a critical threshold concentration of TnpR for resolution. These results show that the responses generated by the chromosomal copy of $\operatorname{luxS}$ are much stronger than external signal molecules for this reporter system.

In vitro perception of the Pectobacterium AI-2 signal by $\mathbf{S a l}$ monella spp. The ability of AI-2-producing cells to alter gene expression in Salmonella spp. was tested by co-inoculating the Salmonella RIVET reporter, in both $l u x S^{+}$and $l u x S^{-}$backgrounds, with wild-type Salmonella or Pectobacterium strains on LB softagar plates. The response of the Salmonella RIVET reporter-only controls showed no difference in resolution between incubation at 37 or $22^{\circ} \mathrm{C}$, indicating that the reporter was active at environmental temperatures (Fig. 5). A significant difference in resolution due to the reporter's luxS genotype was observed for the reporter-only controls; however, the presence of AI-2-producing strains of Salmonella or Pectobacterium was unable to rescue activity of the Salmonella luxS $S^{-}$RIVET reporter back to wild-type lux $S^{+}$levels. Co-culture with the AI-2-producing Pectobacterium strains increased resolution in the $l u x S^{+}$Salmonella reporter; however, the increase was not significant and was not duplicated in the $\operatorname{lux} S^{-}$reporter.

In vivo promoter expression measured via RIVET assays. To determine how soft rots affected the activity of Salmonella luxS, the activity of CEC0026 $\left(\mathrm{P}_{l u x S}\right.$-tnpR-lacZ) was determined in green tomato with Pectobacterium soft rots (Fig. 6A). The reporter was strongly expressed in all samples, indicating that the luxS promoter was highly active in intact and Pectobacterium soft-rotted green tomato fruit. The small reduction of expression in Pectobacterium SR38 soft rots was the result of 3 of 11 samples not reaching $100 \%$ resolution and was not significant as determined by a $t$ test comparison with non-soft-rotted green tomato $(P=0.193)$.
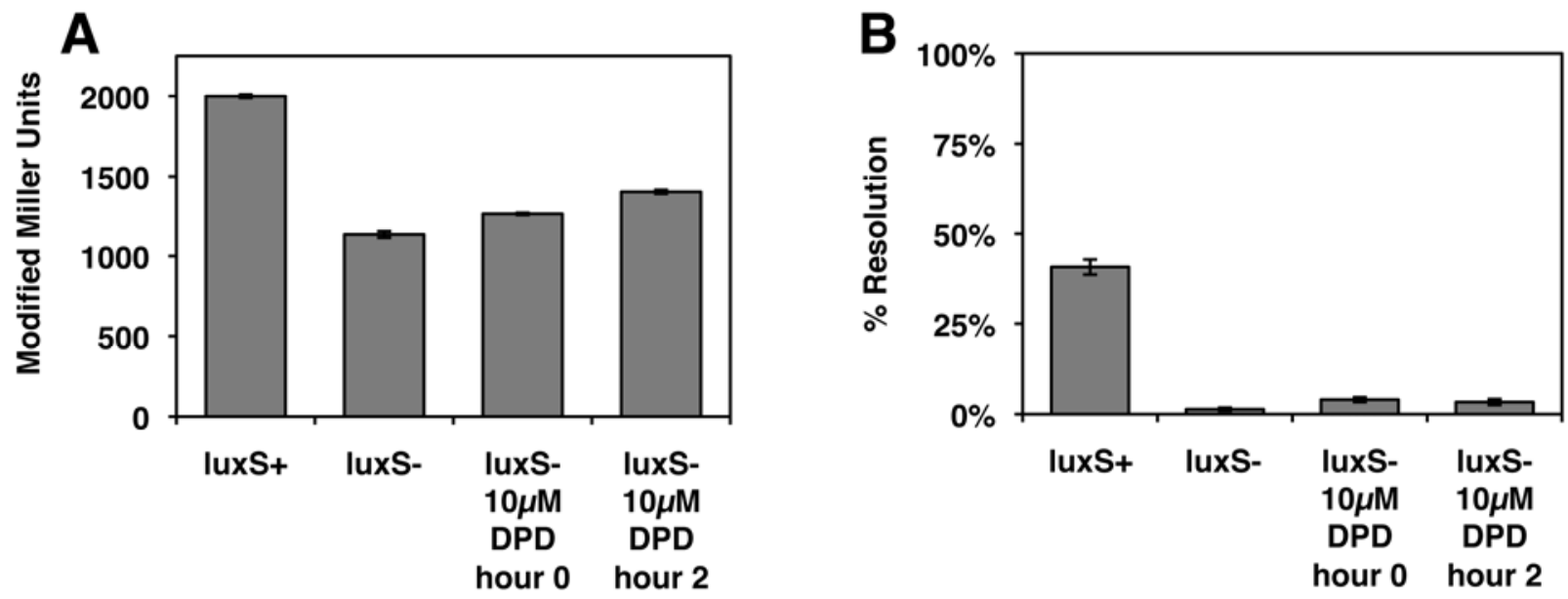

Fig. 4. Activity of the $l s r G::$ tnpR-lacZ reporter in response to synthetic AI-2. A, Expression of the resolvase in vivo expression technology (RIVET) reporter was determined after $24 \mathrm{~h}$ of growth in Luria-Bertani liquid culture at $37^{\circ} \mathrm{C}$. Addition of synthetic AI-2 (10 $\mu \mathrm{M}(\mathrm{S})-4,5$-dihydroxy-2,3-pentandione [DPD] $)$ increased resolution of the lsrG::tnpR-lacZ luxS $S^{-}$reporter (CEC0018) but did not rescue resolution to the level of the luxS ${ }^{+}$reporter (CEC0015). Bars represent standard error of three technical and three biological replicates. B, LacZ activity $8 \mathrm{~h}$ after inoculation. Differences between groups are all significant according to the Tukey-Kramer honestly significant difference test; however, addition of DPD does not fully rescue activity of the luxS ${ }^{\complement}$ background back to the wild-type level. Bars represent standard error of four technical and three biological replicates.
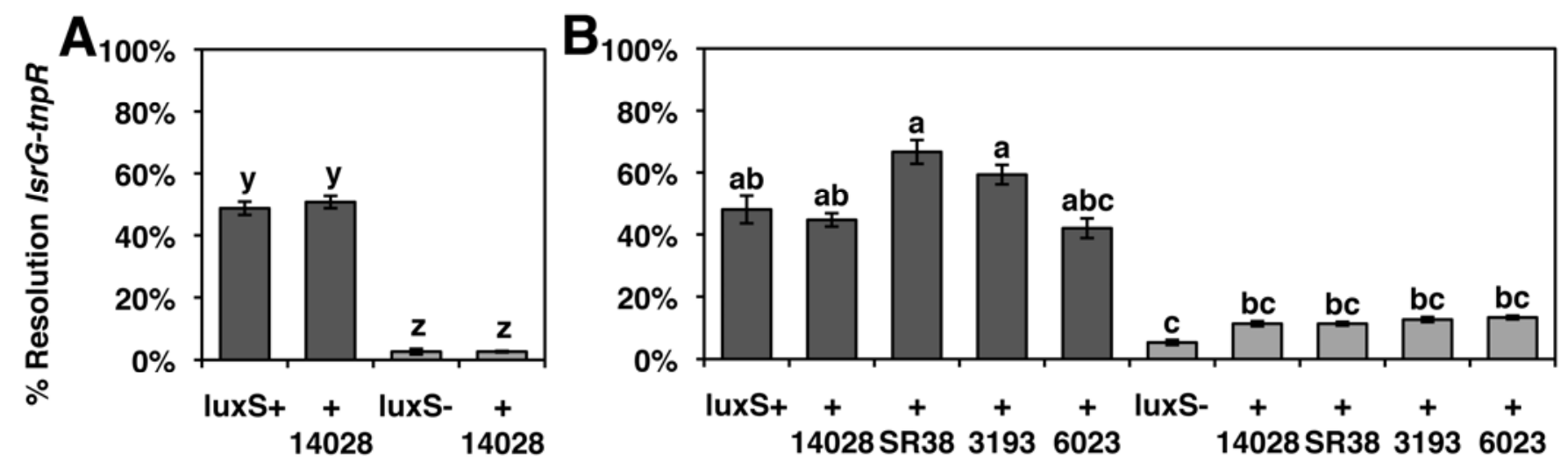

Fig. 5. Resolution of the lsrG::tnpR-lacZ reporter in response to co-culture with Salmonella or Pectobacterium strains SR38 (luxS $S^{+}$), SCC3193 (lux $\left.S^{+}\right)$, and SCC6023 $\left(\right.$ lux $\left.S^{-}\right)$on Luria-Bertani soft agar at $\mathbf{A}, 37^{\circ} \mathrm{C}$ and $\mathbf{B}, 22^{\circ} \mathrm{C}$. Letters represent significance groups assigned by the Tukey-Kramer honestly significant difference test. Differences are only significant for those groups which share no letters in common. Dark bars represent the lux $S^{+}$background reporter (CEC0015) and the light bars represent the luxS $S^{-}$background reporter (CEC0018). Co-cultured strains were mixed 1:1 prior to inoculation of the soft agar. Bars represent standard error of three technical and three biological replicates. 
Expression of $l s r G:: t n p R-l a c Z$ in response to the soft rots in green tomato was dependent on the luxS background of the Salmonella host as well as the Pectobacterium strain which caused the soft rot (Fig. 6B). In non-soft-rotted green tomato $(P<0.001)$ and Pectobacterium SCC3913 $\left(l u x S^{+}\right)$soft-rotted tomato $(P=0.026)$, there was a significant decrease in RIVET resolution in the Salmonella luxS $S^{-}$reporter (CEC0018) compared with the $\operatorname{lux} S^{+}$reporter (CEC0015). The reduction in resolution was of a magnitude similar to that seen between the same reporters due to their luxS backgrounds during the in vitro assays. Soft rots caused by Pectobacterium strains SR38 (lux $\left.S^{+}\right)(P=$ $0.134)$ and SCC6023 $\left(\operatorname{lux} S^{-}\right)(P=0.781)$ showed nonsignificant decreases in resolution between the Salmonella lux $S^{-}$reporter compared with the $\operatorname{lux} \mathrm{S}^{+}$reporter. The lack of significance may be due to the reduction in resolution seen with the $\operatorname{lux} S^{+}$reporter in these soft rots compared with intact green tomato, which reduces the reporter's dynamic range. However, Pectobacterium SR38 soft rots significantly reduced Salmonella RIVET resolution compared with non-soft-rotted tomato regardless of the reporter's luxS genotype, $(P<0.001$ for CEC0015/SR38 and $P=0.002$ for CEC0018/SR38). SCC3913, which is also lux $S^{+}$, had no affect on resolution $(P=0.924$ for CEC005/SCC3913 and $P=0.992$ for CEC0018/SCC3193). Interestingly, the luxS $S^{-}$strain SCC6023 caused a significant reduction in lsrG::tnpR-lac $Z$ expression in lux $S^{+}$Salmonella $(P=0.001$ for green tomato versus SCC6023) and a small, nonsignificant increase in expression in lux $S^{-}$Salmonella $(P=0.537$ for green tomato versus SCC6023). Taken together, these results show that interactions between Pectobacterium and Salmonella spp., which alter Salmonella gene regulation, are influenced by environmental conditions and strain genotype but not AI-2 signal exchange.

PEL activity in response to exogenous AI-2. Although results of the competitive fitness and RIVET assays clearly showed that AI-2 signal exchange does not significantly affect the fitness of Salmonella spp., they also show that unknown interactions which influence Salmonella fitness and gene expression occur under certain circumstances. To test whether these interactions could impact the PEL activity of Pectobacterium spp., synthetic DPD, Salmonella strains, and culture filtrates were incubated with Pectobacterium SR38 under PEL-inducing conditions (Fig. 7). Only culture filtrates from Salmonella spp. grown at $37^{\circ} \mathrm{C}$, which showed low AI-2 activity in the $V$. harveyi assay, significantly $(P=0.003)$ decreased the PEL activity of SR38 in regards to the Pecto- bacterium SR38 control. Because the filtrate was generated from cultures grown in LB, their addition also altered the metabolic conditions of the M9 $1 \%$ pectin induction media. The additional nutrients allowed a slightly faster growth rate and reduced reliance on PEL, which could result in lower PEL activity. These results suggest that nutritive conditions are more relevant to Pectobacterium PEL activity than AI-2-based signal exchange under the conditions tested.

\section{DISCUSSION}

Because the role of AI-2 signaling in Salmonella spp. has primarily been studied in relation to vertebrate hosts, there are few examples of its potential functions in interactions with phytobacteria. A study of fresh produce identified AI-2 activity in

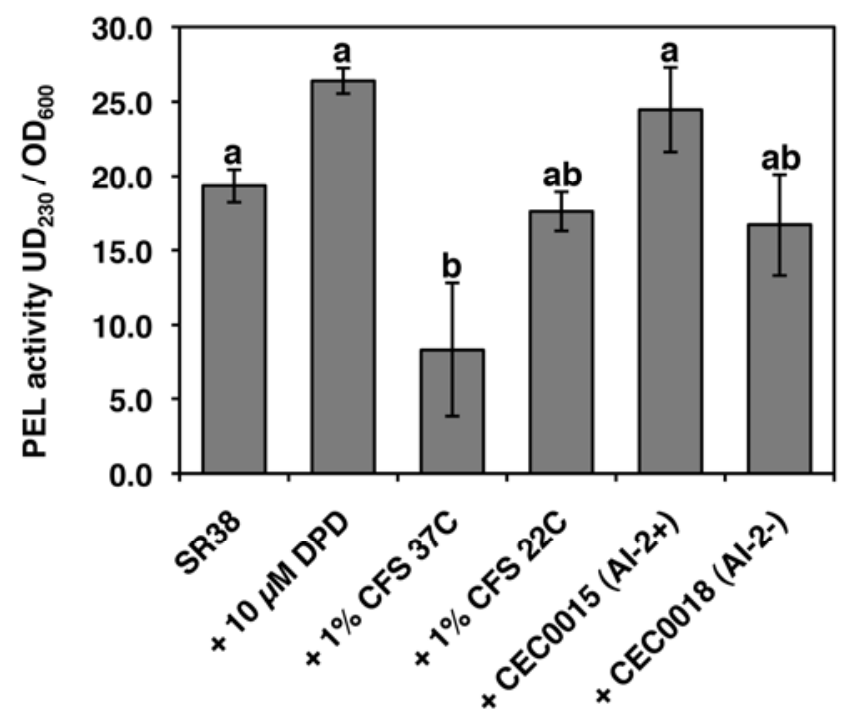

Fig. 7. Pectate lyase activity of Pectobacterium SR38 in response to co-culture with Salmonella spp., synthetic AI-2 ((S)-4,5-dihydroxy-2,3-pentandione), or culture filtrates from Salmonella 14028 cultures grown at 22 or $37^{\circ} \mathrm{C}$. Letters represent significance groups assigned by the Tukey-Kramer honestly significant difference test. Bars represent standard error of three technical and three biological replicates.
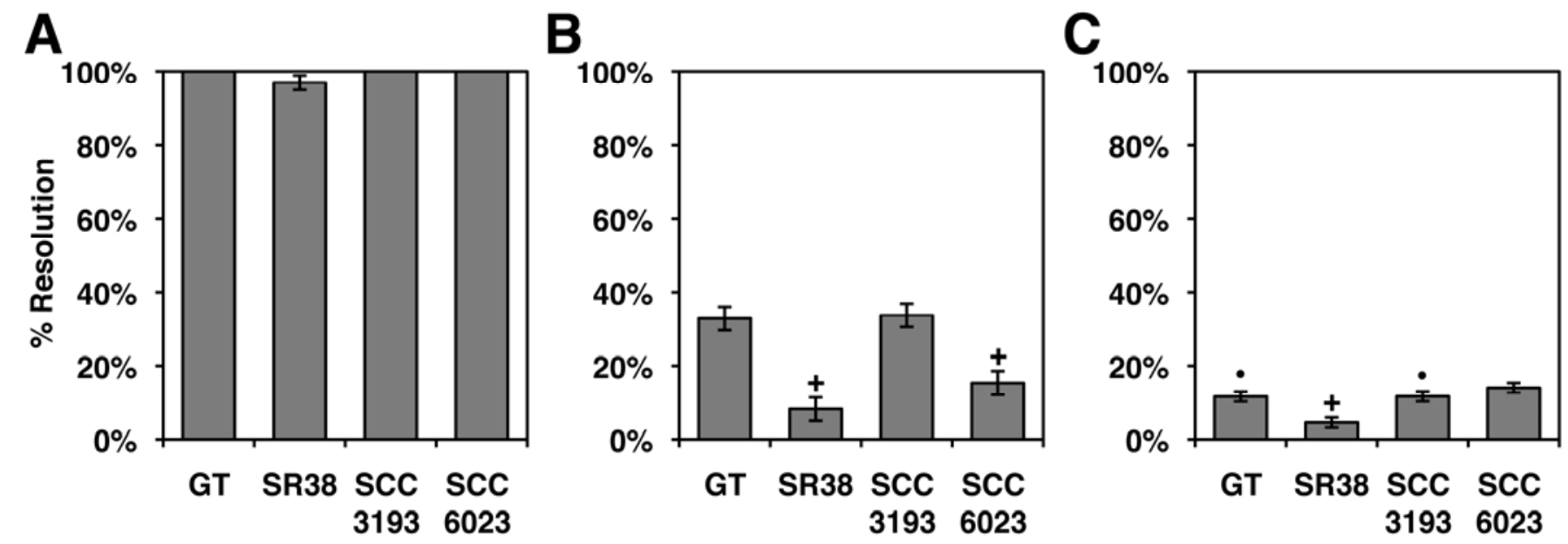

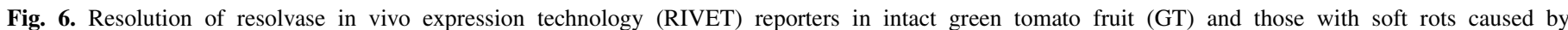

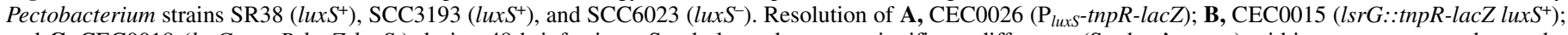

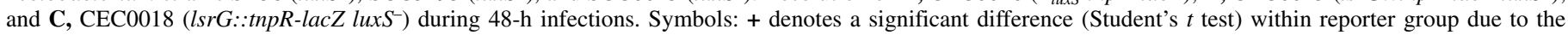

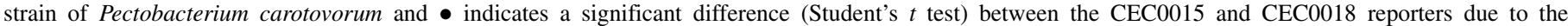

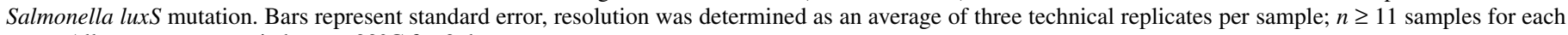
assay. All assays were carried out at $22^{\circ} \mathrm{C}$ for 2 days. 
surface swabs of 11 of the 12 commodities examined, including tomato, indicating that AI-2-based signaling is likely to be widespread in the phyllosphere (29). The AI-2 activity recovered from surface washes during a 9-day study of stored Roma tomato fruit varied independently of the total heterotrophic bacterial count, indicating that the concentration is dynamically controlled by the commensal population or that unculturable members of the phytomicrobiota are responsible for a significant portion of the AI-2 production (29).

The only known previous study to examine competitive fitness phenotypes of AI-2-related mutants of Salmonella in produce found no difference in ultimate population density, growth dynamics, or formation of cell aggregates (an important survival phenotype to resist desiccation in the phyllosphere) between an luxS mutant and wild-type Salmonella spp. during colonization of cilantro leaves (8). The same luxS mutation significantly reduced population densities in the intestines, spleen, and feces during infection of live chicks (8). The luxS mutant utilizes some carbon sources less efficiently compared with the wild type and these differences in the nutrition available to Salmonella spp. in the respective environments are hypothesized to be responsible for the differences in fitness observed for the luxS mutant between colonization of chicks and cilantro leaves (8).

Studies of AI-2-related phenotypes in Salmonella spp. and E. coli have typically used luxS mutants to eliminate AI-2 production. Because LuxS has a dual role in signal production and the degradation of toxic intermediates in the activated methyl cycle, it is difficult to separate effects due to metabolic regulation from those specifically associated with AI-2 signaling during assays which only use an $\operatorname{lux} S$ mutant $(17,23,40,57)$. In this study, the use of an lsrACDBF, "signal-blind" mutant provided an opportunity to observe phenotypes related specifically to the perception of AI-2 signaling without the metabolic side effects of the luxS mutation. However, the competitive fitness of the lsrACDBF mutant was essentially unaltered compared with $S$. enterica 14028 in intact or soft-rotted tomato fruit, indicating that the perception of AI-2 did not significantly affect the fitness of Salmonella spp. The $\operatorname{luxS}$ and $\operatorname{luxS} \operatorname{ls} \mathrm{s} A C D B F$ mutants showed a statistically nonsignificant trend of increased fitness in the presence of Pectobacterium SR38 soft rots in both green FL47 and red Campari tomato, indicating that LuxS may induce changes in Salmonella spp. which influence cross-species interactions. The use of Pectobacterium SCC3192 and its isogenic luxS mutant SCC6023 for competitive fitness assays allowed evaluation of how the Pectobacterium luxS phenotype affected Salmonella survival in soft rots. Both Salmonella luxS and luxS lsrACDBF mutants showed a trend toward increased competitive fitness in SCC6023 (lux $\left.S^{-}\right)$ soft-rotted green tomato fruit, providing additional evidence that LuxS-mediated interactions may occur in soft rots, although it remains unclear how $\operatorname{luxS}$ mutations in both bacteria could increase the fitness of Salmonella spp.

A mutation in $\operatorname{luxS}$ did not affect in vitro growth rate of the Salmonella or Pectobacterium strains in this study. Deletion of luxS is linked to a reduction of motility in a number of species, including Salmonella spp. and P. carotovora $(15,23,25)$. Although motility is linked to plant virulence in $P$. atroseptica, an luxS mutation in $P$. carotovora reduces motility but does not substantially attenuate potato virulence $(15,28,34)$. Similarly, no reduction in the tomato virulence due to an luxS mutation in $P$. carotovorum was observed in this study. The presence of flagella is known to reduce the competitive fitness of Salmonella spp. in planta and a number of motility genes, including those associated with flagella, were identified by microarray as upregulated in wild-type Salmonella spp. compared with an luxS mutant $(24,25,35)$. However, the hypothesis that the effects of $\operatorname{luxS}$ mutation on motility are indirectly responsible for the phenotype of the mutants in soft rots is unlikely to be true because motility genes were shown to be downregulated inside tomato soft rots (21).
The metabolic conditions in ripening tomato fruit are complicated, because the concentrations of at least 60 metabolites constantly change during the ripening process (10). P. carotovorum soft rots are a result of extensive tissue degradation and represent a dynamic environment where the ripening tomato is rapidly degrading into a nutrient-rich liquid, causing additional nutritive alterations. The drastic difference in the development time of Pectobacterium SR38 soft rots between red and green tomato and the inability of Pectobacterium SCC3193 and SCC6023 to consistently reproduce soft rots in red tomato indicated the importance of nutritive and environmental conditions to the establishment of bacteria within the tomato fruit. The difference in observed trends of competitive fitness between the luxS and lsrACDBF luxS mutant of Salmonella in green FL47 and red Campari tomato, although inconclusive, suggest regulation of metabolism by LuxS may also impact Salmonella fitness in tomato.

In general, there were large variations in the competitive index values for individual samples within each Salmonella mutant coinfection group for the intact red tomato and Pectobacterium SR38 soft-rot treatments. However, this level of variability was not observed in the intact green tomato treatment, the JS246 versus 14028 control infection of intact red tomato, or previously reported competitive co-infections with defined mutants of genes identified as active by a promoter probe screen in intact red tomato and, therefore, does not appear to be inherent in the assay (35). Thus, the high variability in treatments other than intact green tomato observed during this study likely indicates that survival in these nutritionally dynamic environments is driven to an important extent by stochastic processes or is a result of interactions of multiple factors. Differing nutritive conditions are known to cause large changes in the LuxS regulon which could contribute to the trends in competitive fitness observed for Salmonella spp. in soft rots $(25,39,59,60)$.

Microarray studies have shown that a majority of luxS-responsive genes are not influenced by the AI-2 signal itself. For example, in Streptococcus mutans, only 9.2\% (59/644) of the genes differentially regulated between the wild type and an luxS mutant responded to exogenous AI-2 (53). In E. coli O157:H7, only $1.9 \%$ of genes (18/951) responded directly to synthetic AI-2 when metabolic effects of the high concentration of DPD (100 $\mu \mathrm{M})$ were controlled (26). Of the 547 genes in the Salmonella LuxS regulon, only 43 genes $(7.9 \%)$ were differentially regulated by both the luxS mutation and the addition of Salmonella culture filtrates with AI-2 activity $(25,64)$. Of those genes, $31(72.1 \%)$ generated a larger response in the luxS mutant, indicating that the presence of luxS, not AI-2 signal exchange, is primarily responsible for the regulatory changes.

In the current study a $\mathrm{P}_{\text {luxs }}$-tnpR-lacZ (CEC0026) RIVET reporter was highly active in intact tomato as well as soft rots. Although culture filtrates taken from wild-type Salmonella cultures grown in $\mathrm{LB}$ at $22^{\circ} \mathrm{C}$ showed increased AI-2 activity compared with those grown at $37^{\circ} \mathrm{C}$, expression of the lsrG-tnpR reporter on LB soft agar was similar at 22 and $37^{\circ} \mathrm{C}$, indicating that the threshold concentration of AI-2 sufficient for regulatory activity was present at both temperatures. Because Salmonella AI2 production is induced by preferred carbon sources (such as glucose), low $\mathrm{pH}$, and high osmolarity, conditions which should all be present in tomato fruit, it is likely that $\operatorname{luxS}$ was expressed and AI-2 produced in tomato fruit under the tested conditions (51). The lsrG::tnpR-lacZ RIVET reporters had similar activity in vitro and in vivo, indicating that the physical environment of tomato fruit was permissive to perception of AI-2. The reporters CEC0015 (lux $\left.S^{+}\right)$and CEC0018 (lux $\left.S^{-}\right)$showed strong differences in resolution in response to their luxS genotype in vitro and during infection of intact green tomato fruit. However, activity of the lux $S^{-}$reporter was not rescued back to wild-type level by incubation with DPD or co-culture with AI-2-producing strains. The inability to rescue an luxS mutation in Salmonella spp. by 
addition of exogenous AI-2 has been frequently reported by others and is believed to be indicative of unknown metabolic factors which affect AI-2-based regulatory changes instead of a problem with reporter function (40).

Interestingly, soft rots caused by Pectobacterium strains SR38 $\left(\operatorname{lux} S^{+}\right)$and SCC6023 $\left(\operatorname{lux} S^{-}\right)$, but not SCC3193 $\left(\operatorname{lux} S^{+}\right)$, repressed resolution of the Salmonella luxS+ RIVET reporter (CEC0015) to the level of the $l u x S^{-}$reporter (CEC0018). SR38 also significantly repressed resolution of CEC0018. However, because SCC6023 lacks luxS it cannot affect resolution via AI-2 signaling. Also, CEC0018 does not supply AI-2 to the surrounding environment, and the perception of AI-2 from SR38 would be expected to increase RIVET resolution. Therefore, the observed the reductions in RIVET resolution do not appear related to AI-2 signaling but may respond to differences in LuxS activity.

The relationship between Salmonella spp. and $P$. carotovorum during colonization of produce continues to be an interesting area of research relevant to produce safety. However, this study shows that, under the conditions tested, these interactions are not significantly influenced by AI-2 signaling and are likely controlled by the nutritive environment of the tomato fruit as well as the metabolic capabilities of each strain, which could be influenced by LuxS.

\section{ACKNOWLEDGMENTS}

This research was supported by the United States Department of Agriculture National Institute of Food and Agriculture (USDA-NIFA) grant 2011-67017-30127 and by the Center for Produce Safety through UCANR/USDA NIFA grant 2010-34608-20768 (SA7660) and the Florida Specialty Crops Foundation. C. E. Cox was supported by a National Science Foundation Graduate Research Fellowship and a USDA-NIFA Post-Doctoral Fellowship, Agriculture and Food Research Initiative Competitive Grant Number 2012-67012-19708, Current Research Information System project FLA-SWS-005175. Additionally, M. McClelland was supported, in part, by National Institutes of Health Contract Number HHSN272200900040C from the Pathosystems Resource Integration Center and grants AI039557 AI052237, AI073971, AI075093, AI077645, and AI083646; USDA grants 2009-03579-30127; and the Binational Agricultural Research and Development Fund. We thank A. Mäe for providing Pectobacterium strains SCC3913 and SCC6023 and K. R. Schneider and G. Hochmuth for graciously providing the green tomato used in this research.

\section{LITERATURE CITED}

1. Anderson, M., Jaykus, L.-A., Beaulieu, S., and Dennis, S. 2011. Pathogen-produce pair attribution risk ranking tool to prioritize fresh produce commodity and pathogen combinations for further evaluation (P3ARRT). Food Control 22:1865-1872.

2. Barak, J. D., Kramer, L. C., and Hao, L. Y. 2011. Colonization of tomato plants by Salmonella enterica is cultivar dependent, and type 1 trichomes are preferred colonization sites. Appl. Environ. Microbiol. 77:498-504.

3. Bartz, J. A. 1988. Potential for postharvest disease in tomato fruit infiltrated with chlorinated water. Plant Dis. 72:9-13.

4. Bassler, B. L., Wright, M., and Silverman, M. R. 1994. Multiple signalling systems controlling expression of luminescence in Vibrio harveyi: sequence and function of genes encoding a second sensory pathway. Mol. Microbiol. 13:273-286.

5. Bauer, W. D., and Mathesius, U. 2004. Plant responses to bacterial quorum sensing signals. Curr. Opin. Plant Biol. 7:429-433.

6. Brandl, M. T. 2006. Fitness of human enteric pathogens on plants and implications for food safety. Annu. Rev. Phytopathol. 44:367-392.

7. Brandl, M. T. 2008. Plant lesions promote the rapid multiplication of Escherichia coli O157:H7 on postharvest lettuce. Appl. Environ. Microbiol. 74:5285-5289.

8. Brandl, M. T., Miller, W. G., Bates, A. H., and Mandrell, R. E. 2005. Production of autoinducer 2 in Salmonella enterica serovar Thompson contributes to its fitness in chickens but not on cilantro leaf surfaces. Appl. Environ. Microbiol. 71:2653-2662.

9. Brown, S. P., Inglis, R. F., and Taddei, F. 2009. Evolutionary ecology of microbial wars: Within-host competition and (incidental) virulence. Evol. Appl. 2:32-39.
10. Carrari, F., Baxter, C., Usadel, B., Urbanczyk-Wochniak, E., Zanor, M.-I., Nunes-Nesi, A., Nikiforova, V., Centero, D., Ratzka, A., Pauly, M., Sweetlove, L. J., and Fernie, A. R. 2006. Integrated analysis of metabolite and transcript levels reveals the metabolic shifts that underlie tomato fruit development and highlight regulatory aspects of metabolic network behavior. Plant Physiol. 142:1380-1396.

11. Centers for Disease Control and Prevention (CDC). 2007. Multistate outbreaks of Salmonella infections associated with raw tomatoes eaten in restaurants-United States, 2005-2006. Morbid. Mortal. Weekly Rep. 56:909-911.

12. Chatterjee, A., Cui, Y., and Chatterjee, A. K. 2002. RsmA and the quorum-sensing signal, N-[3-oxohexanoyl]-L-homoserine lactone, control the levels of rsmB RNA in Erwinia carotovora subsp. carotovora by affecting its stability. J. Bacteriol. 184:4089-4095.

13. Cherepanov, P. P., and Wackernagel, W. 1995. Gene disruption in Escherichia coli: TcR and KmR cassettes with the option of Flp-catalyzed excision of the antibiotic-resistance determinant. Gene 158:9-14.

14. Choi, J., Shin, D., and Ryu, S. 2007. Implication of quorum sensing in Salmonella enterica serovar typhimurium virulence: The luxS gene is necessary for expression of genes in pathogenicity island 1. Infect. Immun. 75:4885-4890.

15. Coulthurst, S. J., Lilley, K. S., and Salmond, G. P. C. 2006. Genetic and proteomic analysis of the role of $\operatorname{luxS}$ in the enteric phytopathogen, Erwinia carotovora. Mol. Plant Pathol. 7:31-45.

16. Datsenko, K. A., and Wanner, B. L. 2000. One-step inactivation of chromosomal genes in Escherichia coli K-12 using PCR products. Proc. Natl. Acad. Sci. USA 97:6640-6645.

17. De Keersmaecker, S. C. J., Sonck, K., and Vanderleyden, J. 2006. Let LuxS speak up in AI-2 signaling. Trends Microbiol. 14:114-119.

18. De Keersmaecker, S. C. J., Varszegi, C., van Boxel, N., Habel, L. W., Metzger, K., Daniels, R., Marchal, K., De Vos, D., and Vanderleyden, J. 2005. Chemical synthesis of (S)-4,5-dihydroxy-2,3-pentanedione, a bacterial signal molecule precursor, and validation of its activity in Salmonella typhimurium. J. Biol. Chem. 280:19563-19568.

19. Fatica, M. K., and Schneider, K. R. 2011. Salmonella and produce: Survival in the plant environment and implications in food safety. Virulence 2:1-7.

20. Franz, E., and van Bruggen, A. H. C. 2008. Ecology of E. coli O157:H7 and Salmonella enterica in the primary vegetable production chain. Crit. Rev. Microbiol. 34:143-161.

21. Goudeau, D. M., Parker, C. T., Zhou, Y., Sela, S., Kroupitski, Y., and Brandl, M. T. 2013. The Salmonella transcriptome in lettuce and cilantro soft rot reveals a niche overlap with the animal host intestine. Appl. Environ. Microbiol. 79(1):250-262.

22. Griffith, K. L., and Wolf, R. E. 2002. Measuring beta-galactosidase activity in bacteria: Cell growth, permeabilization, and enzyme assays in 96-well arrays. Biochem. Biophys. Res. Commun. 290:397-402.

23. Hardie, K. R., and Heurlier, K. 2008. Establishing bacterial communities by "word of mouth": LuxS and autoinducer 2 in biofilm development. Nat. Rev. Microbiol. 6:635-643.

24. Iniguez, A. L., Dong, Y., Carter, H. D., Ahmer, B. M. M., Stone, J. M., and Triplett, E. W. 2005. Regulation of enteric endophytic bacterial colonization by plant defenses. Mol. Plant-Microbe Interact. 18:169-178.

25. Jesudhasan, P. R., Cepeda, M. L., Widmer, K., Dowd, S. E., Soni, K. A., Hume, M. E., Zhu, J., and Pillai, S. D. 2010. Transcriptome analysis of genes controlled by luxS/autoinducer-2 in Salmonella enterica serovar typhimurium. Foodborne Pathogens Dis. 7:399-410.

26. Kendall, M. M., Rasko, D. A., and Sperandio, V. 2007. Global effects of the cell-to-cell signaling molecules autoinducer-2, autoinducer-3, and epinephrine in a luxS mutant of enterohemorrhagic Escherichia coli. Infect. Immun. 75:4875-4884

27. Klerks, M. M., Franz, E., van Gent-Pelzer, M., Zijlstra, C., and van Bruggen, A. H. C. 2007. Differential interaction of Salmonella enterica serovars with lettuce cultivars and plant-microbe factors influencing the colonization efficiency. ISME J. 1:620-631.

28. Laasik, E., Andresen, L., and Mäe, A. 2006. Type II quorum sensing regulates virulence in Erwinia carotovora ssp. carotovora. FEMS Microbiol. Lett. 258:227-234.

29. Lu, L., Hume, M. E., and Pillai, S. D. 2005. Autoinducer-2-like activity on vegetable produce and its potential involvement in bacterial biofilm formation on tomatoes. Foodborne Pathogens Dis. 2:242-249.

30. Matsumoto, H., Muroi, H., Umehara, M., Yoshitake, Y., and Tsuyumu, S. 2003. Peh production, flagellum synthesis, and virulence reduced in Erwinia carotovora subsp. carotovora by mutation in a homologue of cytR. Mol. Plant-Microbe Interact. 16:389-397.

31. Merighi, M., Ellermeier, C. D., Slauch, J. M., and Gunn, J. S. 2005. Resolvase-in vivo expression technology analysis of the Salmonella enterica serovar typhimurium PhoP and PmrA regulons in BALB/c mice. J. Bacteriol. 187:7407-7416.

32. Miller, J. 1972. Experiments in Molecular Genetics. Cold Springs Harbor 
Laboratory Press, Cold Springs Harbor, NY

33. Miller, S. T., Xavier, K. B., Campagna, S. R., Taga, M. E., Semmelhack, M. F., Bassler, B. L., and Hughson, F. M. 2004. Salmonella typhimurium recognizes a chemically distinct form of the bacterial quorum-sensing signal AI-2. Mol. Cell 15:677-687.

34. Mulholland, V., Hinton, J. C., Sidebotham, J., Toth, I. K., Hyman, L. J., Pérombelon, M. C., Reeves, P. J., and Salmond, G. P. 1993. A pleiotropic reduced virulence (Rvi-) mutant of Erwinia carotovora subspecies atroseptica is defective in flagella assembly proteins that are conserved in plant and animal bacterial pathogens. Mol. Microbiol. 9:343-356.

35. Noel, J. T., Arrach, N., Alagely, A., McClelland, M., and Teplitski, M. 2010. Specific responses of Salmonella enterica to tomato varieties and fruit ripeness identified by in vivo expression technology. PloS One 5:e12406

36. Noel, J. T., Joy, J., Smith, J. N., Fatica, M., Schneider, K. R., Ahmer, B. M. M., and Teplitski, M. 2010. Salmonella SdiA recognizes N-acyl homoserine lactone signals from Pectobacterium carotovorum in vitro, but not in a bacterial soft rot. Mol. Plant-Microbe Interact. 23:273-282.

37. Osorio, C. G., Crawford, J. A., Michalski, J., Martinez-Wilson, H., Kaper, J. B., and Camilli, A. 2005. Second-generation recombination-based in vivo expression technology for large-scale screening for Vibrio cholerae genes induced during infection of the mouse small intestine. Infect. Immun. 73:972-980.

38. Parsek, M. R., and Singh, P. K. 2003. Bacterial biofilms: An emerging link to disease pathogenesis. Annu. Rev. Microbiol. 57:677-701.

39. Pereira, C. S., Santos, A. J. M., Bejerano-Sagie, M., Correia, P. B., Marques, J. C., and Xavier, K. B. 2012. Phosphoenolpyruvate phosphotransferase system regulates detection and processing of the quorum sensing signal autoinducer-2. Mol. Microbiol. 84:93-104.

40. Pereira, C. S., Thompson, J. A, and Xavier, K. B. 2012. AI-2-mediated signalling in bacteria. FEMS Microbiol. Rev. doi:10.1111/j.15746976.2012.00345.x

41. Pirhonen, M., Flego, D., Heikinheimo, R., and Palva, E. T. 1993. A small diffusible signal molecule is responsible for the global control of virulence and exoenzyme production in the plant pathogen Erwinia carotovora. EMBO J. 12:2467-2476.

42. Prouty, A. M., Schwesinger, W. H., and Gunn, J. S. 2002. Biofilm formation and interaction with the surfaces of gallstones by Salmonella spp. Infect. Immun. 70:2640-2549.

43. Rezzonico, F., and Duffy, B. 2008. Lack of genomic evidence of AI-2 receptors suggests a non-quorum sensing role for $\operatorname{luxS}$ in most bacteria. BMC Microbiol. 8:154.

44. Rezzonico, F., Smits, T. H. M., and Duffy, B. 2012. Detection of AI-2 receptors in genomes of enterobacteriaceae suggests a role of type-2 quorum sensing in closed ecosystems. Sensors 12:6645-6665.

45. Richards, G. M., and Beuchat, L. R. 2005. Metabiotic associations of molds and Salmonella Poona on intact and wounded cantaloupe rind. Int. J. Food Microbiol. 97:327-339.

46. Schauder, S., Shokat, K., Surette, M. G., and Bassler, B. L. 2001. The LuxS family of bacterial autoinducers: Biosynthesis of a novel quorumsensing signal molecule. Mol. Microbiol. 41:463-476.

47. Smith, J. N., and Ahmer, B. M. M. 2003. Detection of other microbial species by Salmonella: Expression of the SdiA regulon. J. Bacteriol. 185:1357-1366.

48. Smith, J. N., Dyszel, J. L., Soares, J. A., Ellermeier, C. D., Altier, C., Lawhon, S. D., Adams, L. G., Konjufca, V., Curtiss, R., Slauch, J. M., and Ahmer, B. M. M. 2008. SdiA, an N-acylhomoserine lactone receptor, becomes active during the transit of Salmonella enterica through the gastrointestinal tract of turtles. PloS One 3:e2826.

49. Soares, J. A., and Ahmer, B. M. M. 2011. Detection of acyl-homoserine lactones by Escherichia and Salmonella. Curr. Opin. Microbiol. 14:188193.

50. Sun, J., Daniel, R., Wagner-Döbler, I., and Zeng, A.-P. 2004. Is autoinducer-2 a universal signal for interspecies communication: A comparative genomic and phylogenetic analysis of the synthesis and signal transduction pathways. BMC Evol. Biol. 4:36.

51. Surette, M. G., and Bassler, B. L. 1999. Regulation of autoinducer production in Salmonella typhimurium. Mol. Microbiol. 31:585-595.

52. Surette, M. G., Miller, M. B., and Bassler, B. L. 1999. Quorum sensing in Escherichia coli, Salmonella typhimurium, and Vibrio harveyi: A new family of genes responsible for autoinducer production. Proc. Natl. Acad. Sci. USA 96:1639-1644.

53. Sztajer, H., Lemme, A., Vilchez, R., Schulz, S., Geffers, R., Yip, C. Y. Y., Levesque, C. M., Cvitkovitch, D. G., and Wagner-Döbler, I. 2008. Autoinducer-2-regulated genes in Streptococcus mutans UA159 and global metabolic effect of the luxS mutation. J. Bacteriol. 190:401-415.

54. Taga, M. E. 2005. Methods for analysis of bacterial autoinducer-2 production. In: Current Protocols in Microbiology, Chapter 1: Unit 1C.1. John Wiley \& Sons, Hoboken, NJ.

55. Taga, M. E., Miller, S. T., and Bassler, B. L. 2003. Lsr-mediated transport and processing of AI-2 in Salmonella typhimurium. Mol. Microbiol. 50:1411-1427.

56. Taga, M. E., Semmelhack, J. L., and Bassler, B. L. 2001. The LuxSdependent autoinducer AI-2 controls the expression of an ABC transporter that functions in AI-2 uptake in Salmonella typhimurium. Mol. Microbiol. 42:777-793.

57. Vendeville, A., Winzer, K., Heurlier, K., Tang, C. M., and Hardie, K. R. 2005. Making "sense" of metabolism: Autoinducer-2, LuxS and pathogenic bacteria. Nat. Rev. Microbiol. 3:383-396.

58. Von Bodman, S. B., Bauer, W. D., and Coplin, D. L. 2003. Quorum sensing in plant-pathogenic bacteria. Annu. Rev. Phytopathol. 41:455482.

59. Walters, M., and Sperandio, V. 2006. Quorum sensing in Escherichia coli and Salmonella. Int. J. Med. Microbiol. 296:125-131.

60. Wang, L., Li, J., March, J. C., Valdes, J. J., and Bentley, W. E. 2005. luxSdependent gene regulation in Escherichia coli $\mathrm{K}-12$ revealed by genomic expression profiling. J. Bacteriol. 187:8350-8360.

61. Warriner, K., and Namvar, A. 2010. The tricks learnt by human enteric pathogens from phytopathogens to persist within the plant environment. Current Opin. Biotechnol. 21:131-136.

62. Waters, C. M., and Bassler, B. L. 2005. Quorum sensing: Cell-to-cell communication in bacteria. Annu. Rev. Cell Dev. Biol. 21:319-346.

63. Wells, J. M., and Butterfield, J. E. 1997. Salmonella contamination associated with bacterial soft rot of fresh fruits and vegetables in the marketplace. Plant Dis. 81:867-872.

64. Widmer, K. W., Jesudhasan, P. R., Dowd, S. E., and Pillai, S. D. 2007. Differential expression of virulence-related genes in a Salmonella enterica serotype typhimurium luxS mutant in response to autoinducer AI-2 and poultry meat-derived AI- 2 inhibitor. Foodborne Pathogens Dis. 4:5-15.

65. Williams, P. 2007. Quorum sensing, communication and cross-kingdom signalling in the bacterial world. Microbiology 153:3923-3938.

66. Yamazaki, A., Li, J., Hutchins, W. C., Wang, L., Ma, J., Ibekwe, A. M., and Yang, C.-H. 2011. Commensal effect of pectate lyases secreted from Dickeya dadantii on proliferation of Escherichia coli O157:H7 EDL933 on lettuce leaves. Appl. Environ. Microbiol. 77:156-162. 